Supporting Information

\title{
Bimetallic Two-dimensional Nanoframes: High Activity Acidic Bifunctional Oxygen Reduction and Evolution Electrocatalysts
}

Fernando Godínez-Salomón, ${ }^{1}$ Luis Albiter, ${ }^{1}$ Rubén Mendoza-Cruz ${ }^{2}$ and Christopher P. Rhodes ${ }^{1, *}$

${ }^{1}$ Department of Chemistry and Biochemistry, Texas State University San Marcos Texas 78666, USA

${ }^{2}$ Department of Biomedical Engineering, University of Texas at San Antonio, San Antonio, Texas 78249, USA

${ }^{*}$ Corresponding author: Tel.: 512.245.6721, Fax: 512.245.2374, e-mail address: cprhodes@txstate.edu 

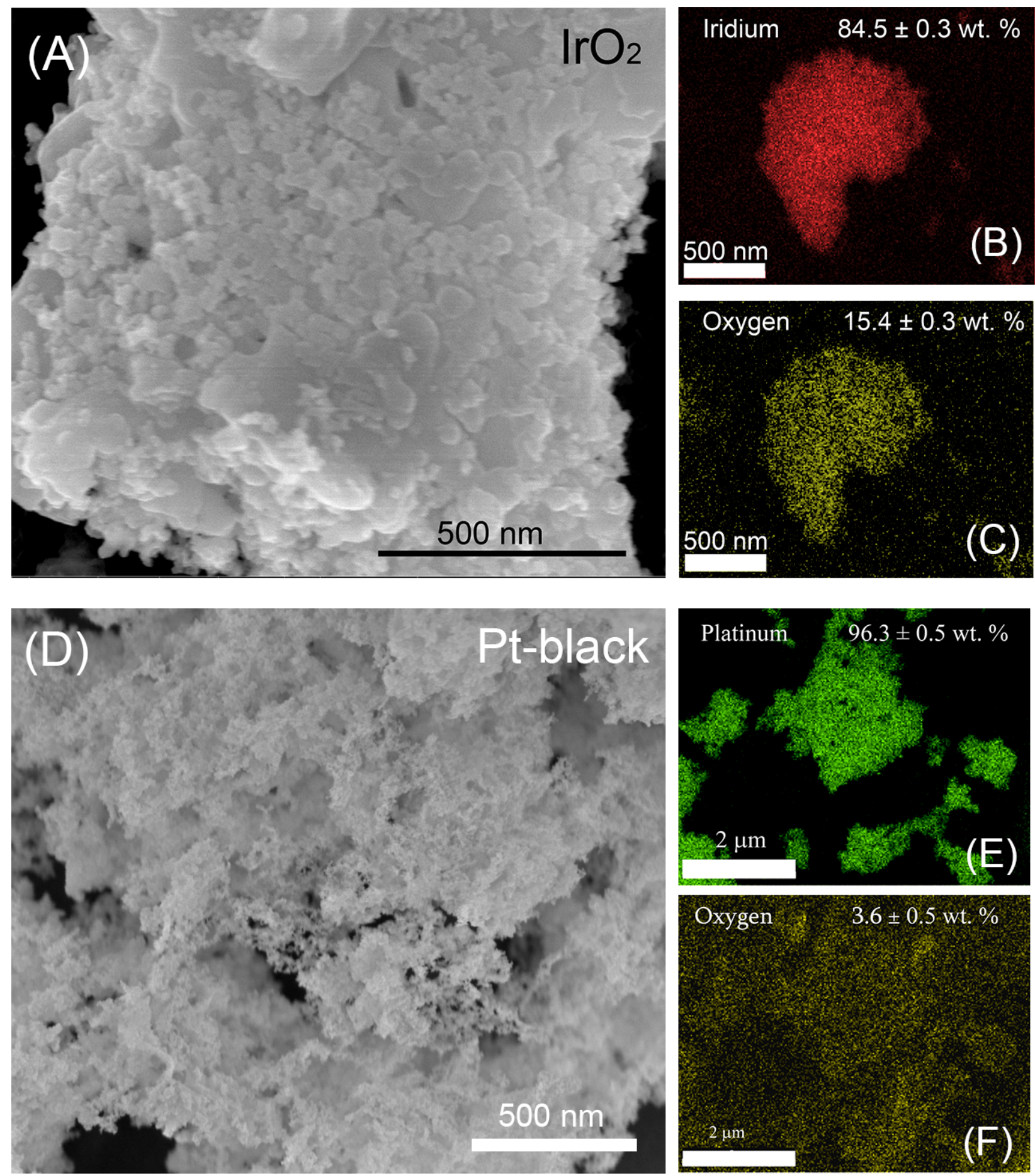

Figure S1. Scanning electron microscopy (SEM) and Energy Dispersive X-ray Spectroscopy (EDS) mapping of commercial $\mathrm{IrO}_{2}$ (Alfa) (A-C) and Pt-black (D-F). 

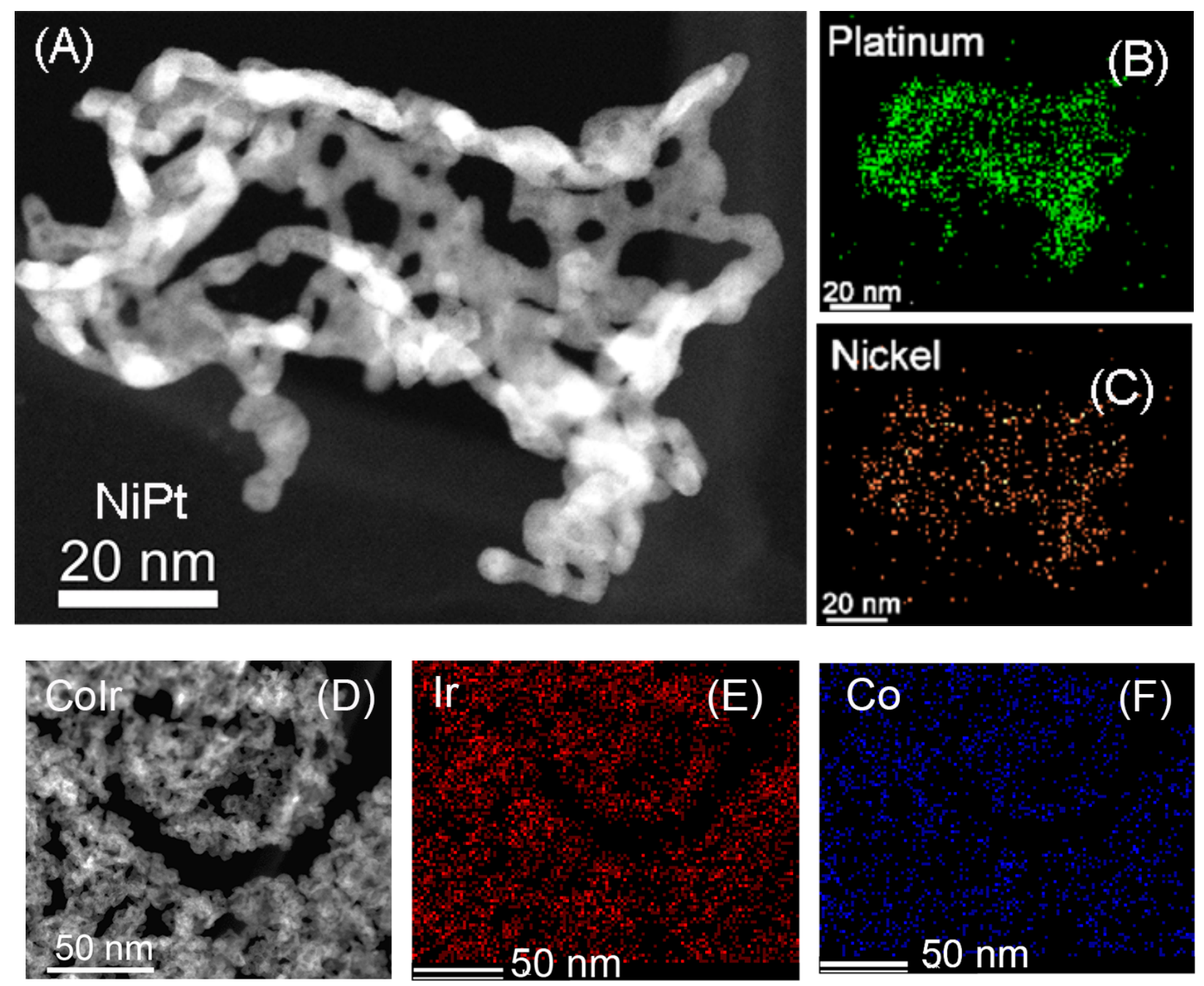

Figure S2. High-angle annular dark-field scanning transmission electron microscopy (HAADFSTEM) image and Energy Dispersive X-ray Spectroscopy (EDS) mapping of NirPt (A-C) and CoIr (D-F) 2D-nanoframes.

Table S1. Relative elemental composition of Pt, Ir, Ni and Co within catalysts and precursors determined by Energy Dispersive X-ray Spectroscopy (EDS).

\begin{tabular}{|l|c|c|c|c|}
\hline Sample & Pt wt. \% & Ir wt. \% & Ni wt. \% & Co wt. \% \\
\hline Pt-black & $96.3 \pm 0.5$ & - & - & - \\
\hline $\mathrm{IrO}{ }_{2}$ & - & $84.5 \pm 0.3$ & - & - \\
\hline $\mathrm{Pt}-\mathrm{NiO}$ & $16.3 \pm 2.7$ & - & $40.9 \pm 7.1$ & - \\
\hline $\mathrm{Ir}-\mathrm{Co}(\mathrm{OH})_{2}$ & - & $23.1 \pm 3.5$ & - & $23.1 \pm 3.5$ \\
\hline $\mathrm{NiPt}-200$ & $31.3 \pm 1.3$ & - & $56.7 \pm 2.9$ & - \\
\hline $\mathrm{CoIr}-300$ & - & $36.0 \pm 1.8$ & - & $46.0 \pm 2.9$ \\
\hline $\mathrm{NiPt}-200_{\mathrm{CL}}(\mathrm{NiPt})$ & $89.1 \pm 2.2$ & - & $5.9 \pm 1.6$ & - \\
\hline $\mathrm{CoIr}-300_{\mathrm{CL}}(\mathrm{CoIr})$ & - & $88.3 \pm 1.5$ & - & $5.2 \pm 0.6$ \\
\hline
\end{tabular}




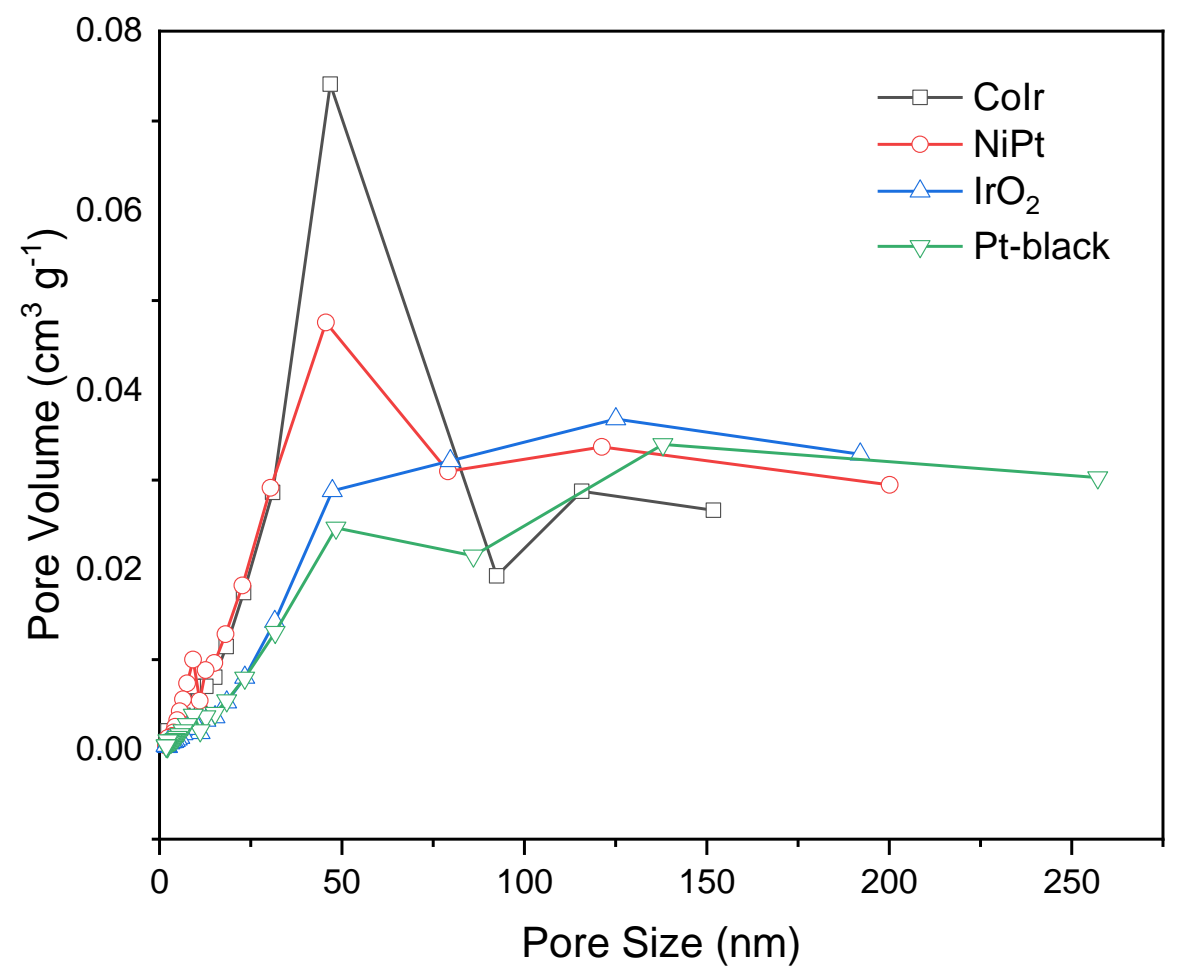

Figure S3. Pore-size distribution (PSD) plot showing cumulative pore volume versus pore size of $\mathrm{NiPt}$, CoIr, Pt black and $\mathrm{IrO}_{2}$ obtained from nitrogen physisorption analysis.

Electrochemical surface areas of NiPt, CoIr, Pt black, and IrO2. Electrochemical surface areas (ECSAs) of the individual components, NiPt, CoIr, Pt black, and $\mathrm{IrO}_{2}$, were determined by either CO-stripping or pseudocapacitance measurements (Table S2). The ECSAs of $\mathrm{NiPt}$, Pt black, and $\mathrm{IrO}_{2}$ are lower in comparison with the BET surface areas determined by nitrogen physisorption (Table 1), but are similar for CoIr. The differences between ECSAs and BET surface areas may be due to a number of possible factors. The presence of Ni within the nearsurface of NiPt can suppress ECSA surface area since CO-adsorption takes place selectively on noble metals. ${ }^{1}$ The presence of non-noble species within the near surface was recently reported by our group for a NiIr catalyst after a similar chemical leaching and electrochemical conditioning process. ${ }^{2}$ Also, micropores within the structure can prevent access of the electrolyte and hinder the electrochemical reaction. ${ }^{3-4}$ 
Table S2. Electrochemical surface area (ECSA) of individual NiPt, CoIr and commercial Pt black and $\mathrm{IrO}_{2}$ (Alfa) electrodes determined from either electrochemical carbon monoxide (CO) stripping voltammetry (notated with “*”) or pseudocapacitance measurements (notated with “**”).

\begin{tabular}{|c|c|}
\hline Material & ECSA $\left(\mathbf{m}^{\mathbf{2}} \mathbf{g}^{-\mathbf{1}}\right)$ \\
\hline Pt-black & $14 \pm 1^{*}$ \\
\hline $\mathrm{NiPt}$ & $27 \pm 1^{*}$ \\
\hline $\mathrm{IrO}_{2}(\mathrm{Alfa})$ & $25 \pm 2^{* *}$ \\
\hline $\mathrm{CoIr}$ & $57 \pm 7^{*}$ \\
\hline
\end{tabular}

\section{Comparison of Cyclic Voltammograms of Combined Materials and Individual Components}

Figure S4,A-B shows the cyclic voltammograms (CVs) of electrodes of Pt-black and $\mathrm{IrO}_{2}$, respectively, the individual constituents of $\mathrm{Pt}-\mathrm{IrO}_{2}$ electrodes. The $\mathrm{CVs}$ show typical voltammetric features of reversible oxidation/reduction processes on metallic $\mathrm{Pt}$ and $\mathrm{IrO}_{2}$ surfaces. ${ }^{2,5}$ In comparison, the experimental CV of $\mathrm{Pt}-\mathrm{IrO}_{2}$ electrodes (Figure S4D), which are composed of the physical mixture of $\mathrm{Pt}$ and $\mathrm{IrO}_{2}$, shows clear differences in the hydrogen adsorption/desorption features $\left(\mathrm{H}_{\text {upd }}\right)$ on Pt compared with features observed within the CV of Pt-black (Figure S4A). Specifically, the hydrogen adsorption/desorption on $\mathrm{Pt}$ were surpressed within $\mathrm{Pt}-\mathrm{IrO}_{2}$ electrodes compared with Pt-black. To futher explore this phenomenon, the currents normalized to the electrode geometric area for the individual CVs of Pt-black (Figure S4A) and $\mathrm{IrO}_{2}$ (Figure S4B) were arthimetically combined, as shown in Figure S4C. The arthimetically combined CV (Figure $\mathrm{S} 4 \mathrm{C}$ ) shows characteristics associated to both $\mathrm{Pt}$ and $\mathrm{IrO}_{2}$ phases, as expected. Unlike Figure $\mathrm{S} 4 \mathrm{C}$, the experimental CV of $\mathrm{Pt}-\mathrm{IrO}_{2}$ (Figure S4D) did not show defined $\mathrm{H}_{\text {upd }}$ features, but rather showed a small broad peak at $\sim 0.3 \mathrm{~V}_{\mathrm{RHE}}$ and a large reversible peak at $\sim 0.9 \mathrm{~V}_{\mathrm{RHE}}$. The differences between the hydrogen adsorption/desorption features observed between the CVs of the experimental Pt$\mathrm{IrO}_{2}$ electrodes and the CVs of the individual components Pt-black and $\mathrm{IrO}_{2}$ supports that the $\mathrm{H}_{\text {upd }}$ features are altered within $\mathrm{Pt}-\mathrm{IrO}_{2}$ electrodes. Similar suppression of $\mathrm{H}_{\text {upd }}$ features on $\mathrm{Pt}-\mathrm{IrO}_{2}$ electrodes has been observed within previosly reported studies; ${ }^{6-7}$ however, the specific reason of these effects have not been clearly determined. We also note that the CO-stripping peaks of Pt$\mathrm{IrO}_{2}$ electrodes (Figure 5A) showed differences compared with $\mathrm{CO}$-stripping peaks of Pt-black (Figure 5B) further supporting changes in the surface within $\mathrm{Pt}-\mathrm{IrO}_{2}$ electrodes compared with Ptblack.

A similar analysis was made to compare the CVs of electrodes of the individual NiPt (Figure S4E) and CoIr (Figure S4F) components with the experimental CV of NiPt-CoIr (Figure $\mathrm{S} 3 \mathrm{H}$ ) and the arthimetically combined CV of NiPt and CoIr (Figure S4G). In contrast with $\mathrm{Pt}_{-} \mathrm{IrO}_{2}$, for NiPt-CoIr the current and qualitative features observed within the experimental curve of the 
physicallly combined NiPt-CoIr electrodes (Figure S4H) were similar to that of the arithmetically combined curve (Figure S4G) obtained by addition of the separate curves of the indidivual components. We attribute the observed differences between the $\mathrm{CVs}$ of $\mathrm{Pt}-\mathrm{IrO}_{2}$ and $\mathrm{NiPt}-\mathrm{CoIr}$ to changes in the adsorption/desorption of protons onto Pt caused by the interparticle contact of $\mathrm{Pt}$ nanoparticles with $\mathrm{IrO}_{2}$ nanoparticles within $\mathrm{Pt}-\mathrm{IrO}_{2}$ which does not occur as significantly within $\mathrm{NiPt}-\mathrm{CoIr}$ due to the integrated architecture that results in less interaction between NiPt and CoIr. 

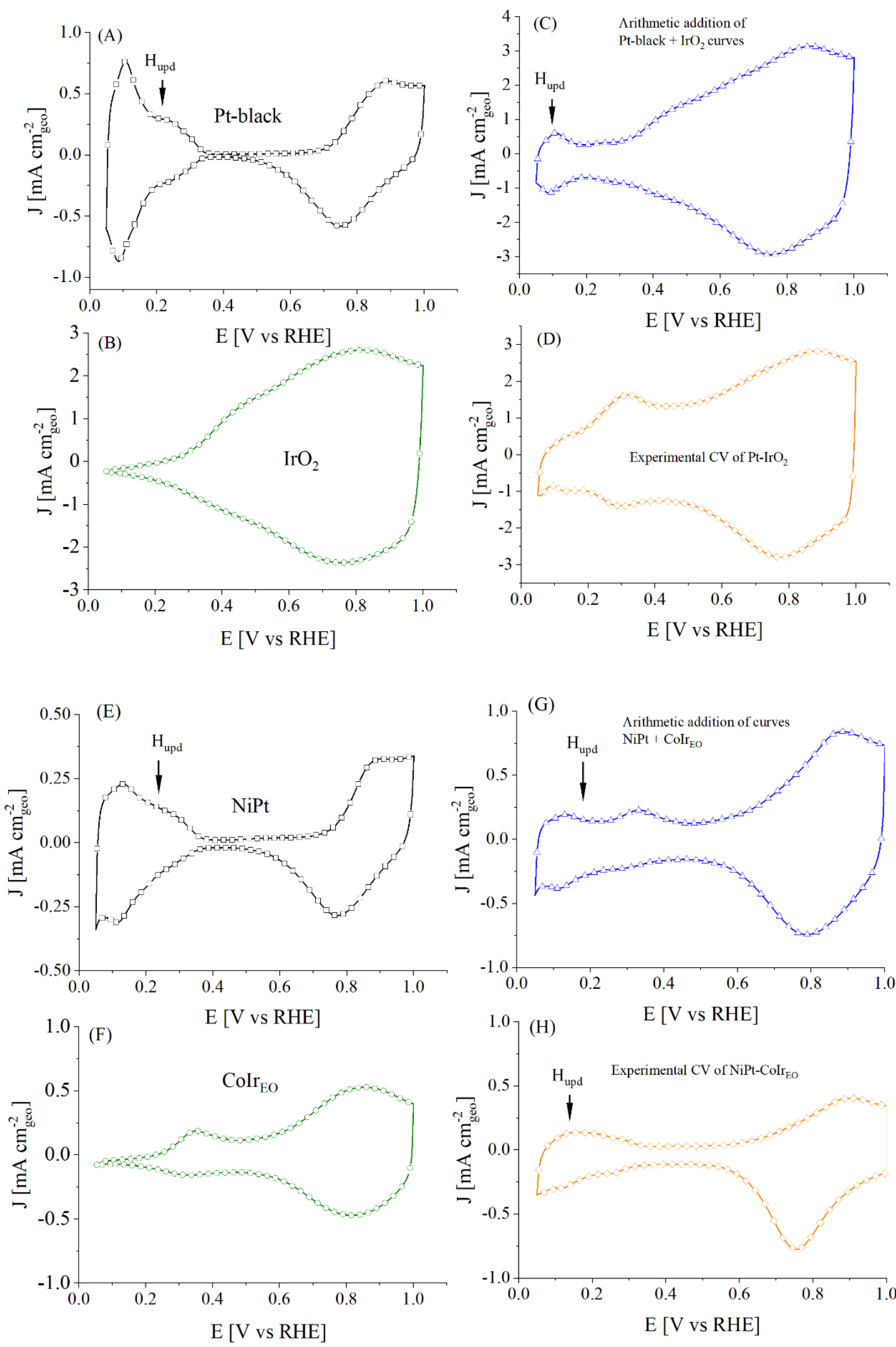

Figure S4. Cyclic voltammetry of bifunctional catalysts constituents Pt-black (A), $\mathrm{IrO}_{2}(\mathrm{~B}), \mathrm{NiPt}$ $(\mathrm{E})$, CoIr-EO (F), the arithmetic additions of Pt-Black $+\mathrm{IrO}_{2}(\mathrm{C}), \mathrm{NiPt}+\mathrm{CoIr}_{\mathrm{EO}}(\mathrm{G})$ and experimental curves of $\mathrm{Pt}-\mathrm{IrO}_{2}(\mathrm{D})$ and $\mathrm{NiPt}-\mathrm{CoIr}_{\mathrm{EO}}(\mathrm{H})$. The suffix "EO" means the catalysts were previously electrochemical oxidized by cycling 60 times between $0.05 \mathrm{~V}_{\mathrm{RHE}}$ to $1.6 \mathrm{~V}_{\mathrm{RHE}}$ in Arsaturated $0.1 \mathrm{M} \mathrm{HClO}_{4}$. 


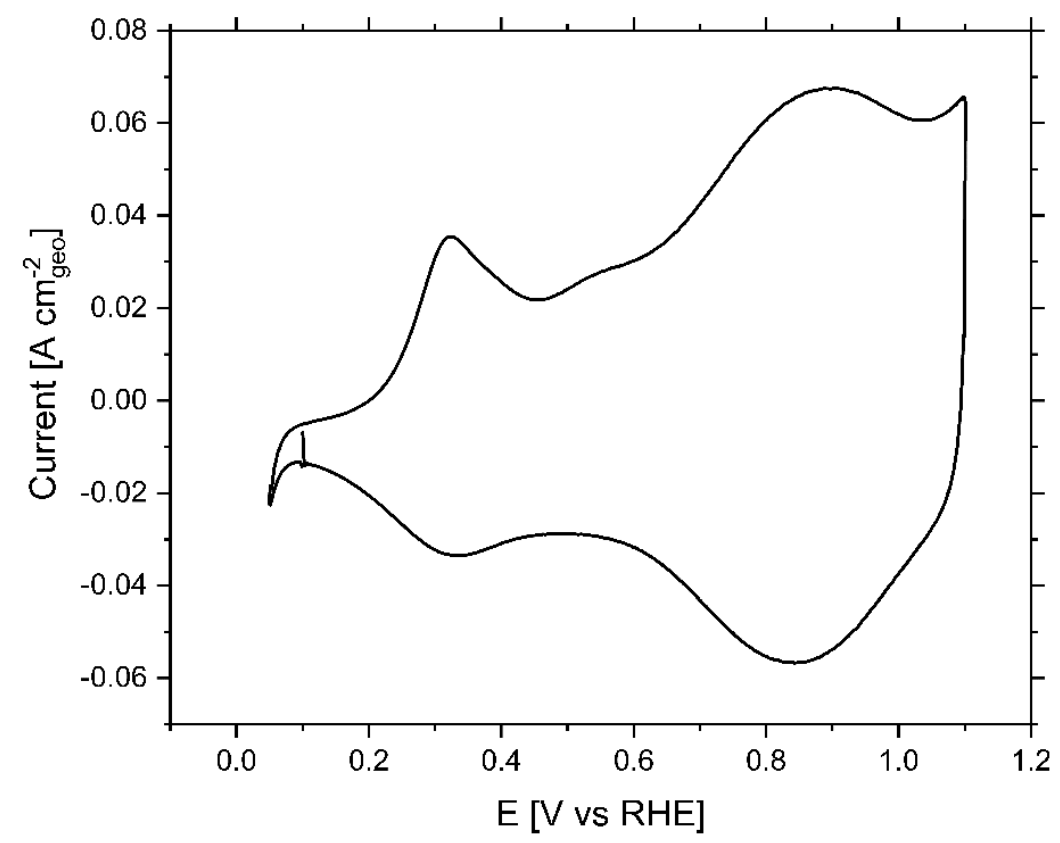

Figure S5. Carbon monoxide (CO)-stripping voltametric scan of CoIr $\operatorname{Ir}_{\mathrm{EO}}$ (after electrochemical oxidation procedure). 

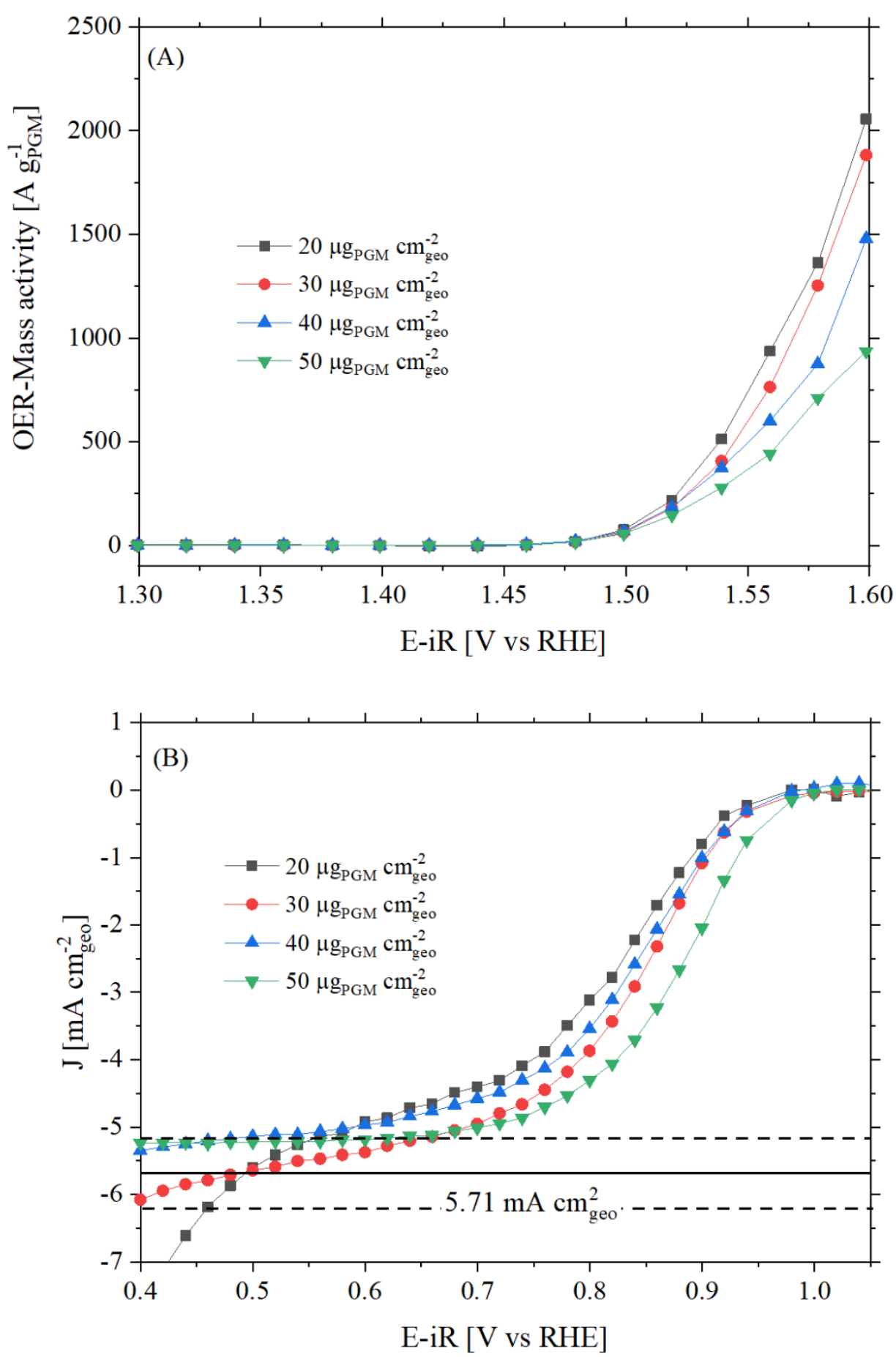

Figure S6. Effect of electrode loading on current in the (A) oxygen evolution reaction (OER) region obtained from chronoamperometry measurements, and (B) oxygen reduction reaction (ORR) region from potentiodynamic measurements for NiPt-CoIr catalysts; data obtained under $\mathrm{O}_{2}$-saturated $0.1 \mathrm{M} \mathrm{HClO}_{4}$ at $1600 \mathrm{rpm}$; the solid black line within (B) represents the theoretical diffusion limiting ORR current for a four electron transfer process, and dotted lines represent $\pm 10 \%$ of the theoretical diffusion limiting current. 
Table S3. Comparison of electrode loadings, electrochemical surface areas (ECSA), oxygen reduction reaction (ORR) and oxygen evolution reaction (OER) mass and specific activities of Pt$\mathrm{IrO}_{2}$ and NiPt-CoIr after electrochemical oxidation (EO) and accelerated durability testing (ADT); the ORR specific activities of were determined by normalizing the ECSA of Pt determined by COstripping and after background and ohmic drop correction using the froward CV curve at a scan rate of $20 \mathrm{mVs}^{-1}$ under $\mathrm{O}_{2}$-saturated $0.1 \mathrm{M} \mathrm{HClO}_{4}$ at $1600 \mathrm{rpm}$; the $\mathrm{OER}$ mass activities were normalized for the mass of $\mathrm{Ir}$ and were determined at $1.51 \mathrm{~V}_{\mathrm{RHE}}$ and $1.55 \mathrm{~V}_{\mathrm{RHE}}$ from chronoamperometry measurements after ohmic drop correction. Percent loss for initial and final ORR and OER mass activities was determined by the ratios of the mass activities after EO and after ADT.

\begin{tabular}{|c|c|c|c|c|}
\hline Category & Parameter & Parameter & $\mathrm{Pt}-\mathrm{IrO}_{2}$ & NiPt-CoIr \\
\hline \multirow{3}{*}{ Loading } & PGM loading & $\left(\mu \mathrm{g}_{\mathrm{PGM}} \mathrm{cm}^{-2} \mathrm{geo}\right)$ & 50 & 50 \\
\hline & Pt loading & $\left(\mu \mathrm{g}_{\mathrm{Pt}} \mathrm{cm}^{-2} \mathrm{geo}\right)$ & $25.6 \pm 1.0$ & $25.4 \pm 0.8$ \\
\hline & Ir loading & $\left(\mu \mathrm{g}_{\mathrm{Ir}} \mathrm{cm}^{-2}\right.$ geo $)$ & $24.7 \pm 2.5$ & $24.4 \pm 0.8$ \\
\hline \multirow{10}{*}{$\begin{array}{c}\text { After } \\
\text { Electrochemical } \\
\text { Oxidation (EO) }\end{array}$} & \multirow[t]{4}{*}{ ORR } & $\mathrm{J}_{\mathrm{geo}} 0.9 \mathrm{~V}\left(\mathrm{~mA} \mathrm{~cm}_{\mathrm{geo}}^{-2}\right)$ & $2.21 \pm 0.43$ & $2.93 \pm 0.50$ \\
\hline & & $\mathrm{J}_{\mathrm{m}}{ }^{0.9 \mathrm{~V}}\left(\mathrm{~A} \mathrm{~g}_{\mathrm{PGM}}{ }^{-1}\right)$ & $77.4 \pm 16.5$ & $139.3 \pm 13.5$ \\
\hline & & $\mathrm{J}_{\mathrm{m}}^{0.9 \mathrm{~V}}\left(\mathrm{~A} \mathrm{~g}_{\mathrm{Pt}^{-1}}{ }^{-1}\right)$ & $143 \pm 30$ & $249.7 \pm 27$ \\
\hline & & $\mathrm{J}_{\mathrm{S}}^{0.9 \mathrm{~V}}\left(\mathrm{~mA} \mathrm{~cm}{ }^{-2} \mathrm{Pt}\right)$ & $1.36 \pm 0.37$ & $0.85 \pm 0.14$ \\
\hline & \multirow[t]{6}{*}{ OER } & $\mathrm{J}_{\mathrm{geo}}{ }^{1.51 \mathrm{~V}}\left(\mathrm{~mA} \mathrm{~cm}^{-2}{ }_{\text {geo }}\right)$ & $0.80 \pm 0.07$ & $7.3 \pm 1.0$ \\
\hline & & $\mathrm{J}_{\mathrm{m}}^{1.51 \mathrm{~V}}\left(\mathrm{~A} \mathrm{~g}_{\mathrm{PGM}}{ }^{-1}\right)$ & $16.8 \pm 1.5$ & $163 \pm 19$ \\
\hline & & $\mathrm{J}_{\mathrm{m}}{ }^{1.51 \mathrm{~V}}\left(\mathrm{~A} \mathrm{~g}_{\mathrm{II}}{ }^{-1}\right)$ & $35 \pm 4$ & $309 \pm 44$ \\
\hline & & 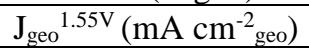 & $4.5 \pm 0.8$ & $30 \pm 1$ \\
\hline & & $\mathrm{J}_{\mathrm{m}}^{1.55 \mathrm{~V}}\left(\mathrm{~A} \mathrm{~g}_{\mathrm{PGM}^{-1}}\right)$ & $95 \pm 16$ & $563 \pm 14$ \\
\hline & & $\mathrm{J}_{\mathrm{m}}^{1.55 \mathrm{~V}}\left(\mathrm{~A} \mathrm{~g}_{\mathrm{II}}^{-1}\right)$ & $201 \pm 46$ & $1300 \pm 72$ \\
\hline \multirow{10}{*}{$\begin{array}{c}\text { After } \\
\text { Accelerated } \\
\text { Durability } \\
\text { Testing (ADT) }\end{array}$} & \multirow[t]{4}{*}{ ORR } & $\mathrm{J}_{\mathrm{geo}} 0.9 \mathrm{~V}\left(\mathrm{~mA} \mathrm{~cm}{ }_{\text {disk }}^{-2}\right)$ & $1.4 \pm 0.2$ & $1.55 \pm 0.41$ \\
\hline & & $\mathrm{J}_{\mathrm{m}}{ }^{0.9 \mathrm{~V}}\left({\left.\mathrm{~A} \mathrm{~g}_{\mathrm{PGM}}{ }^{-1}\right)}\right.$ & $44 \pm 7$ & $44.6 \pm 11.2$ \\
\hline & & 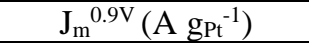 & $89 \pm 13$ & $93 \pm 35$ \\
\hline & & $\mathrm{J}_{\mathrm{S}}^{0.9 \mathrm{~V}}\left(\mathrm{~mA} \mathrm{~cm}{ }^{-2} \mathrm{Pt}\right)$ & $0.78 \pm 0.23$ & $0.89 \pm 0.21$ \\
\hline & \multirow[t]{6}{*}{ OER } & $\mathrm{J}_{\mathrm{geo}}{ }^{1.51 \mathrm{~V}}\left(\mathrm{~mA} \mathrm{~cm}^{-2}{ }_{\mathrm{geo}}\right)$ & $0.43 \pm 0.34$ & $4.9 \pm 0.5$ \\
\hline & & $\mathrm{J}_{\mathrm{m}}{ }^{1.51 \mathrm{~V}}\left(\mathrm{~A} \mathrm{~g}_{\mathrm{PGM}}{ }^{-1}\right)$ & $10.0 \pm 6.8$ & $104.0 \pm 11.9$ \\
\hline & & $\mathrm{J}_{\mathrm{m}}{ }^{1.51 \mathrm{~V}}\left({\left.\mathrm{~A} \mathrm{~g}_{\mathrm{Ir}}{ }^{-1}\right)}\right)$ & $23 \pm 10$ & $222 \pm 30$ \\
\hline & & $\mathrm{J}_{\mathrm{geo}}{ }^{1.55 \mathrm{~V}}\left(\mathrm{~mA} \mathrm{~cm}^{-2} \mathrm{geo}\right)$ & $3.1 \pm 0.7$ & $23.4 \pm 3.1$ \\
\hline & & $\mathrm{J}_{\mathrm{m}}{ }^{1.55 \mathrm{~V}}\left({\left.\mathrm{~A} \mathrm{~g}_{\mathrm{PGM}}{ }^{-1}\right)}\right.$ & $67 \pm 22$ & $496 \pm 62$ \\
\hline & & $\mathrm{J}_{\mathrm{m}}{ }^{1.55 \mathrm{~V}}\left(\mathrm{~A} \mathrm{~g} \mathrm{~g}_{\mathrm{Ir}}^{-1}\right)$ & $123 \pm 52$ & $961 \pm 152$ \\
\hline
\end{tabular}


Table S4. Comparison of the geometric and mass activities for the ORR and OER of electrocatalysts tested in this study and previously reported values. "N/A" corresponds to not available.

\begin{tabular}{|c|c|c|c|c|c|c|}
\hline \multirow[b]{2}{*}{ Material } & \multirow[b]{2}{*}{$\begin{array}{c}\text { Loading } \\
\left(\mu \mathbf{g}_{\mathrm{PGM}}\right. \\
\left.\mathbf{c m}^{-2}{ }_{\text {geo }}\right)\end{array}$} & \multicolumn{2}{|c|}{ ORR (at $0.85 V_{\text {RHE}}$ ) } & \multicolumn{2}{|c|}{ OER (at $1.55 V_{\text {RHE }}$} & \multirow[b]{2}{*}{ Ref. } \\
\hline & & $\mathbf{j} / \mathbf{m}_{\substack{\text { geo } \\
\text { ma }}}^{\mathbf{~} \mathbf{c m}^{-}}$ & $\mathrm{j} / \mathbf{m A ~ m g}^{-1} \mathrm{Pt}$ & $\mathrm{j} / \mathbf{m A ~ c m}^{-2}{ }_{\text {geo }}$ & $\mathbf{j} / \mathbf{m A} \mathbf{~ m g}^{-1}{ }_{I r}$ & \\
\hline NiPt-CoIr ${ }^{\mathrm{a}}$ & 50 & $3.66 \pm 0.63$ & $778 \pm 103$ & $30 \pm 1$ & $1300 \pm 72$ & \\
\hline $\mathrm{Pt}-\mathrm{IrO}_{2}{ }^{\mathrm{a}}$ & 50 & $3.11 \pm 0.49$ & $207 \pm 28$ & $4.5 \pm 0.8$ & $201 \pm 46$ & - \\
\hline Pt-IrO $1: 9^{\mathrm{b}}$ & 380 & 1.64 & 107.4 & 8.42 & 25.0 & 8 \\
\hline $\mathrm{Pt}-\mathrm{Ir} / \mathrm{IrO}_{2}-\mathrm{PtO}_{2}{ }^{\mathrm{b}}$ & 340 & N/A & 27.4 & N/A & $47^{\mathrm{c}}$ & 9 \\
\hline $\mathrm{Pt}-\mathrm{Ir} / \mathrm{IrO}_{2}-\mathrm{PtO}_{2}{ }^{\mathrm{b}}$ & 113 & N/A & 21.7 & N/A & 42.3 & 10 \\
\hline $\mathrm{Pt}-\mathrm{IrO}_{2}{ }^{\mathrm{b}}$ & 380 & 2.3 & 35 & 4.7 & 24.5 & 11 \\
\hline Pt-IrO ${ }_{x}^{b}$ & 380 & 1.3 & 9.8 & 11.2 & 60.4 & 11 \\
\hline $\mathrm{Pt} / \mathrm{s}-\mathrm{IrO}_{2}{ }^{\mathrm{c}}$ & N/A & - & 38.9 & - & 10.6 & 11 \\
\hline $\mathrm{Pt} / \mathrm{IrO}_{2}{ }^{\mathrm{d}-\mathrm{c}}$ & N/A & N/A & 0.31 & N/A & 5.4 & 7 \\
\hline PtIr/rGO*d-c & 145 & 3.0 & N/A & 7.2 & N/A & 12 \\
\hline $\mathrm{Pt}_{3} \mathrm{Ir} / \mathrm{TiCN}^{\mathrm{b}}$ & 61 & NR & 2.8 & NR & 26 & 13 \\
\hline PtIr ${ }^{\mathrm{b}-\mathrm{c}}$ & 510 & 1.0 & 2.2 & 11.2 & 146 & 14 \\
\hline $\mathrm{Pt} / \mathrm{Ir}-\mathrm{IrO}{ }_{2}$ & 340 & N/A & 5.8 & NR & 82 & 15 \\
\hline
\end{tabular}

${ }^{a}$ Chronoamperometry, $0.1 \mathrm{M} \mathrm{HClO}_{4}, 1600 \mathrm{rpm}$, iR-corrected

${ }^{\mathrm{b}} \mathrm{LSV}, 5 \mathrm{mVs}^{-1}, 0.5 \mathrm{M} \mathrm{H}_{2} \mathrm{SO}_{4}, 1600 \mathrm{rpm}$.

${ }^{\mathrm{c}}$ Calculated after digitalization of corresponding figure, using OriginPro $2019 \mathrm{~b}$.

${ }^{d} \mathrm{LSV}, 0.1 \mathrm{M} \mathrm{HClO}_{4}, 0.5-50 \mathrm{mV} / \mathrm{s}, 1600-2500 \mathrm{rpm}$, iR-corrected, no-background subtraction

* rGO: Reduced graphene oxide

Table S5. Comparison of loading, ORR voltage ( $\mathrm{V}_{\text {ORR }}$ vs RHE), OER voltage ( $\mathrm{V}_{\text {OER VS RHE), and }}$ round trip efficiency $\left(\varepsilon_{\mathbf{R T}}\right)$ at $1.5 \mathrm{~mA} \mathrm{~cm}^{-2}$ geo for NiPt-CoIr and $\mathrm{Pt}-\mathrm{IrO}_{2}$, as well as comparative data from literature; equation used for round trip efficiency calculation is described in the text.

\begin{tabular}{|c|c|c|c|c|c|}
\hline Material & $\begin{array}{c}\text { Loading } \\
\left(\mu \mathbf{g}_{\mathbf{P G M}} \mathbf{c m}^{-}\right. \\
\left.{ }_{\mathbf{g e o}}\right)\end{array}$ & $\begin{array}{l}\text { V }_{\text {ORR }}{ }^{a} \\
\left(V_{\text {RHE }}\right)\end{array}$ & $\begin{array}{l}\text { V }_{\text {OER }}{ }^{a} \\
\left(\mathbf{V}_{\text {RHE }}\right)\end{array}$ & $\varepsilon_{\mathrm{RT}}(\%)$ & Reference \\
\hline NiPt-CoIr & 50 & 0.91 & 1.48 & $61.1 \pm 0.6$ & \multirow{2}{*}{ N/A } \\
\hline Pt-IrO 2 & 50 & 0.89 & 1.52 & $58.3 \pm 0.1$ & \\
\hline $\mathrm{Pt}_{85} \mathrm{Ir}_{15} / \mathrm{TiO}_{2}$ & 100 & 0.830 & 1.504 & 55.2 & 16 \\
\hline $\mathrm{PtIr} / \mathrm{Ti}_{4} \mathrm{O}_{7}$ & NR & 0.889 & 1.500 & 59.3 & 17 \\
\hline $\begin{array}{l}\text { Pt-Ir alloy nano- } \\
\text { short-chains }\end{array}$ & 41 & 0.70 & 1.50 & 46.7 & 18 \\
\hline $\mathrm{Pt} / \mathrm{C}$ & N/A & 0.859 & 1.590 & 54.0 & 17 \\
\hline
\end{tabular}

${ }^{a}$ Values at $1.5 \mathrm{~mA} \mathrm{~cm}^{-2}$ geo 
Table S6. Comparison of percent mass and dissolution rates of $\mathrm{Pt}$ and $\mathrm{Ir}$ within $\mathrm{Pt}-\mathrm{IrO}_{2}$ and NiPtCoIr electrodes after electrochemical oxidation (EO) and after accelerated durability testing (ADT) determined from ICP-MS analysis of electrolyte and initial mass on electrode; additional details are provided in the main text.

\begin{tabular}{|c|c|c|c|c|}
\hline Catalyst & Element & $\begin{array}{c}\text { Analysis after } \\
\text { electrochemical } \\
\text { oxidation (EO) }\end{array}$ & \multicolumn{2}{|c|}{$\begin{array}{c}\text { Analysis after accelerated } \\
\text { durability testing (ADT) }\end{array}$} \\
\cline { 3 - 5 } & & $\begin{array}{c}\text { Percent mass } \\
\text { (wt \%), } \\
\text { dissolved in } \\
\text { electrolyte }\end{array}$ & $\begin{array}{c}\text { Percent mass } \\
\text { (wt \%), } \\
\text { dissolved in } \\
\text { electrolyte }\end{array}$ & $\begin{array}{c}\text { dissolution rate } \\
\left(\mathbf{n g} \text { cm }^{-2} \text { cycle }^{-1} \text { ) }\right.\end{array}$ \\
\hline $\mathrm{Pt}^{-I r O}{ }_{2}$ & $\mathrm{Pt}$ & 3.7 & 15.5 & 18.9 \\
\cline { 2 - 5 } & $\mathrm{Ir}$ & 0.2 & 0.2 & 0.3 \\
\hline \multirow{2}{*}{$\mathrm{NiPt}-\mathrm{CoIr}$} & $\mathrm{Pt}$ & 6.2 & 5.3 & 4.7 \\
\cline { 2 - 5 } & $\mathrm{Ir}$ & 4.9 & 1.0 & 0.8 \\
\hline
\end{tabular}



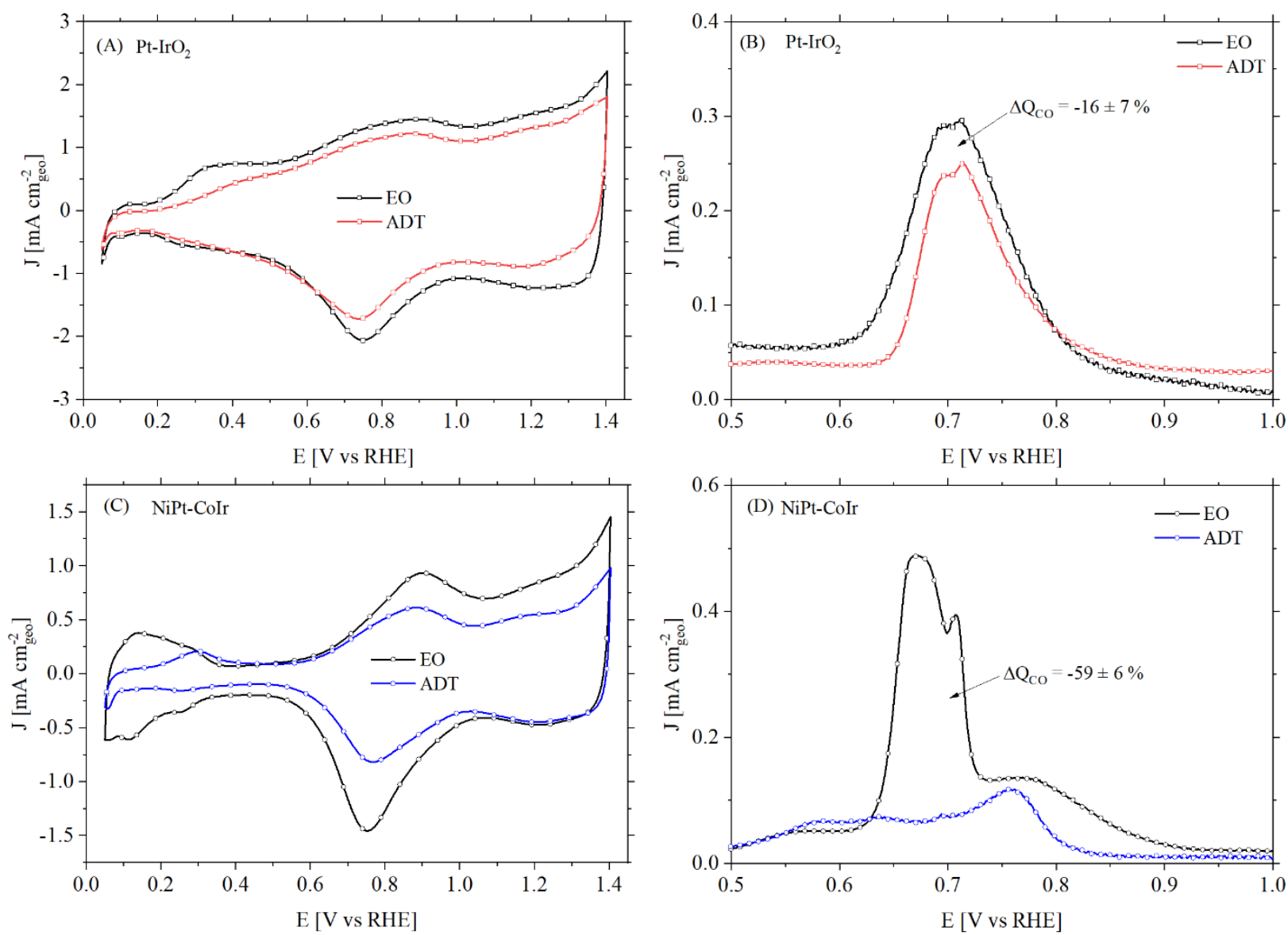

Figure S7. Cyclic voltammetric (A, C) and carbon monoxide (CO) stripping curves (B, D) of Pt$\mathrm{IrO}_{2}$ and NiPt-CoIr before and after accelerated durability testing (ADT). The degradation protocol consisted on scanning 1,000 times under ORR-OER potentials $\left(0.1 \leq \mathrm{E} \leq 1.6 \mathrm{~V}_{\mathrm{RHE}}\right)$.

\section{References}

(1) Gauthier, Y.; Schmid, M.; Padovani, S.; Lundgren, E.; Bus, V.; Kresse, G.; Redinger, J.; Varga, P., Adsorption Sites and Ligand Effect for Co on an Alloy Surface: A Direct View. Physical Review Letters 2001, 87.

(2) Godinez-Salomon, F.; Mendoza-Cruz, R.; Arellano-Jimenez, M. J.; Jose-Yacaman, M.; Rhodes, C. P., Metallic Two-Dimensional Nanoframes: Unsupported Hierarchical NickelPlatinum Alloy Nanoarchitectures with Enhanced Electrochemical Oxygen Reduction Activity and Stability. ACS Appl. Mater. Interfaces 2017, 9, 18660-18674.

(3) Padgett, E.; Andrejevic, N.; Liu, Z. Y.; Kongkanand, A.; Gu, W. B.; Moriyama, K.; Jiang, Y.; Kumaraguru, S.; Moylan, T. E.; Kukreja, R.; Muller, D. A., Connecting Fuel Cell Catalyst Nanostructure and Accessibility Using Quantitative Cryo-Stem Tomography. J. Electrochem. Soc. 2018, 165, F173-F180.

(4) Sneed, B. T.; Cullen, D. A.; Mukundan, R.; Borup, R. L.; More, K. L., Ptco Cathode Catalyst Morphological and Compositional Changes after Pem Fuel Cell Accelerated Stress Testing. J. Electrochem. Soc. 2018, 165, F3078-F3084.

(5) Godínez-Salomón, F.; Albiter, L.; Alia, S. M.; Pivovar, B. S.; Camacho-Forero, L. E.; Balbuena, P. B.; Mendoza-Cruz, R.; Arellano-Jimenez, M. J.; Rhodes, C. P., Self-Supported 
Hydrous Iridium-Nickel Oxide Two-Dimensional Nanoframes for High Activity Oxygen Evolution Electrocatalysts. ACS Catal. 2018, 8, 10498-10520.

(6) da Silva, G. C.; Mayrhofer, K. J. J.; Ticianelli, E. A.; Cherevko, S., Dissolution Stability: The Major Challenge in the Regenerative Fuel Cells Bifunctional Catalysis. J. Electrochem. Soc. 2018, 165, F1376-F1384.

(7) Yao, W.; Yang, J.; Wang, J.; Nuli, Y., Chemical Deposition of Platinum Nanoparticles on Iridium Oxide for Oxygen Electrode of Unitized Regenerative Fuel Cell. Electrochem. Commun. 2007, 9, 1029-1034.

(8) da Silva, G. C.; Fernandes, M. R.; Ticianelli, E. A., Activity and Stability of Pt/IrO $\mathrm{I}_{2}$ Bifunctional Materials as Catalysts for the Oxygen Evolution/Reduction Reactions. ACS Catal. 2018, 8, 2081-2092.

(9) Kong, F. D.; Liu, J.; Ling, A. X.; Xu, Z. Q.; Shi, M. J.; Kong, Q. S.; Wang, H. Y., Overlapping Structure of Platinum-Iridium Oxide Layers and Its Electrocatalytic Behavior on Bifunctional Oxygen Electrode. Catal. Commun. 2017, 90, 19-22.

(10) Kong, F. D.; Zhang, S.; Yin, G. P.; Zhang, N.; Wang, Z. B.; Du, C. Y., Preparation of Pt/Ir-X(Iro2)(10-X) Bifunctional Oxygen Catalyst for Unitized Regenerative Fuel Cell. J. Power Sources 2012, 210, 321-326.

(11) Kong, F. D.; Liu, J.; Ling, A. X.; Xu, Z. Q.; Wang, H. Y.; Kong, Q. S., Preparation of $\mathrm{IrO}_{2}$ Nanoparticles with SBA-15 Template and Its Supported Pt Nanocomposite as Bifunctional Oxygen Catalyst. J. Power Sources 2015, 299, 170-175.

(12) Kim, I. G.; Nah, I. W.; Oh, I. H.; Park, S., Crumpled Rgo-Supported Pt-Ir Bifunctional Catalyst Prepared by Spray Pyrolysis for Unitized Regenerative Fuel Cells. J. Power Sources 2017, 364, 215-225.

(13) Roca-Ayats, M.; Garcia, G.; Galante, J. L.; Pena, M. A.; Martinez-Huerta, M. V., Electrocatalytic Stability of Ti Based-Supported $\mathrm{Pt}_{3} \mathrm{Ir}$ Nanoparticles for Unitized Regenerative Fuel Cells. Int. J. Hydrog. Energy 2014, 39, 5477-5484.

(14) Jung, H. Y.; Park, S.; Popov, B. N., Electrochemical Studies of an Unsupported Ptir Electrocatalyst as a Bifunctional Oxygen Electrode in a Unitized Regenerative Fuel Cell. J. Power Sources 2009, 191, 357-361.

(15) Kong, F. D.; Zhang, S.; Yin, G. P.; Wang, Z. B.; Du, C. Y.; Chen, G. Y.; Zhang, N., Electrochemical Studies of $\mathrm{Pt} / \mathrm{Ir}-\mathrm{IrO}_{2}$ Electrocatalyst as a Bifunctional Oxygen Electrode. Int. J. Hydrog. Energy 2012, 37, 59-67.

(16) Huang, S. Y.; Ganesan, P.; Jung, H. Y.; Popov, B. N., Development of Supported Bifunctional Oxygen Electrocatalysts and Corrosion-Resistant Gas Diffusion Layer for Unitized Regenerative Fuel Cell Applications. J. Power Sources 2012, 198, 23-29.

(17) Won, J. E.; Kwak, D. H.; Han, S. B.; Park, H. S.; Park, J. Y.; Ma, K. B.; Kim, D. H.; Park, K. W., PtIr/Ti ${ }_{4} \mathrm{O}_{7}$ as a Bifunctional Electrocatalyst for Improved Oxygen Reduction and Oxygen Evolution Reactions. J. Catal. 2018, 358, 287-294.

(18) Zhang, T.; Li, S. C.; Zhu, W.; Zhang, Z. P.; Gu, J.; Zhang, Y. W., Shape-Tunable PtIr Alloy Nanocatalysts with High Performance in Oxygen Electrode Reactions. Nanoscale 2017, 9, 1154-1165. 